\title{
STRUCTURE-BASED PREDICTIVE MODEL FOR COAL CHAR COMBUSTION
}

QUARTERLY REPORT Start Date: 04/01/1999

End Date: 06/30/1999

\author{
Authors: Robert H. Hurt \\ Eric M. Suuberg
}

DOE Award No.: DE-FG22-96PC96249--11

\author{
Brown University \\ Division of Engineering Box D \\ Providence, RI 02912-9104
}




\section{Disclaimer}

This report was prepared as an account of work sponsored by an agency of the United States Government. Neither the United States Government nor any agency thereof, nor any of their employees, makes any warranty, express of implied, or assumes any legal liability or responsibility for the accuracy, completeness, or usefulness of any information, apparatus, product, or process disclosed, or represents that its use would not infringe privately owned rights. Reference herein to any specific commercial product, process, or service by trade name, trademark, manufacturer, or otherwise does not necessarily constitute or imply its endorsement, recommendation, or favoring by the United States Government or any agency thereof. The views and opinions of authors expressed herein do not necessarily state or reflect those of the United States Government or any agency thereof. 


\section{TABLE OF CONTENTS}

EXECUTIVE SUMMARY

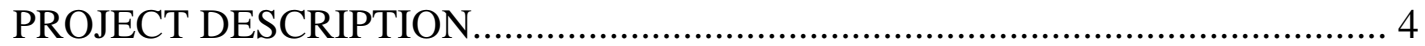

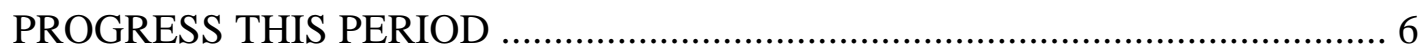

Brief Summary of Activities ............................................................ 6

Detailed Discussions of Progress

Section 1: Surface Anchoring in the Formation 7 of Carbon Structures

Section 2: Computational Chemistry 11

Section 3: Combustion Experiments in Contrasting Flame Types

MILESTONE PLAN attached

MILESTONE LOG attached 


\section{EXECUTIVE SUMMARY}

This report is part on the ongoing effort at Brown University and Ohio State University to develop structure based models of coal combustion. A very fundamental approach is taken to the description of coal chars and their reaction processes, and the results are therefore expected to have broad applicability to the spectrum of carbon materials of interest in energy technologies.

This quarter, our work on structure development in carbons continued continued. A combination of hot stage in situ and ex situ polarized light microscopy was used to identify the preferred orientational of graphene layers at gas interfaces in pitches used as carbon material precursors. The experiments show that edge-on orientation is the equilibrium state of the gas / pitch interface, implying that basal-rich surfaces have higher free energies than edge-rich surfaces in pitch. This result is in agreement with previous molecular modeling studies and TEM observations in the early stages of carbonization. The results may have important implications for the design of tailored carbons with edge-rich or basal-rich surfaces.

In the computational chemistry task, we have continued our investigations into the reactivity of large aromatic rings. The role of $\mathrm{H}$-atom abstraction as well as radical addition to monocyclic aromatic rings has been examined, and a manuscript is currently being revised after peer review. We have also shown that $\mathrm{OH}$ radical is more effective than $\mathrm{H}$ atom in the radical addition process with monocyclic rings. We have extended this analysis to $\mathrm{H}$-atom and $\mathrm{OH}$-radical addition to phenanthrene. Work on combustion kinetics focused on the theoretical analysis of the data previously gathered using thermogravametric analysis. 


\section{PROJECT DESCRIPTION}

The problem of excessive unburned carbon in fly ash could be better managed if designers and users of combustion systems could determine the reactivity of a given char from basic coal properties, avoiding the need to resort to expensive full-scale testing. Establishing a mechanistic link between coal properties and fuel behavior has long been a goal of the coal research community, as such a capability would find numerous uses in predictive tools and optimization tools for coal technologies. Such a predictive capability will not likely be achieved through incremental improvements to current models - new, more fundamental approaches are needed such as the structure-based approach, which we believe has the long term potential to make the required mechanistic links between coal properties and char behaviors.

The overall objective of this project is to carry out the fundamental research needed to develop a first-generation, structure-based model of coal char combustion. The project involves combustion experimentation at a variety of scales, theoretical treatments of surface chemistry, and the development and refinement of advanced modeling techniques describing solid-state transformations in coal chars. The fundamental modeling approaches taken here may also produce auxiliary benefits for other coal technologies, including cokemaking, liquefaction, activated carbon production and use, and carbon materials manufacture (fibers, composites, graphite, etc.). The crystalline structure of carbons and its evolution during processing plays an important role in each of these diverse applications.

This combined experimental and theoretical approach will result in a first-generation, structurebased model that is a significant improvement over empirical models in its ability to:

- predict the rank-dependence of char reactivity

- predict the dependence of char reactivity on heat treatment conditions

- describe reaction kinetics in a wide variety of combustion environments

\section{Task Structure}

This Project consists of the following three interrelated tasks:

\section{Task 1. Project Management}

This task involves reporting, documentation, coordination of effort at the three participating universities, and interactions with the advisory board.

\section{Task 2. Development of Structure-Based Models}

The objective of this is the development of new models that describe the combustion process on a more fundamental basis. Dynamic models will be formulated that describe the evolution of char crystal structures in flames, and fundamental computational treatments of oxidative attack on model $\mathrm{PAH}$ and graphitic structures will be carried out. This task also includes laboratory-scale 
experiments designed to establish link between char structure and oxidation reactivity, and a direct investigation of carbon crystalline rearrangements by in- situ, hot-stage HRTEM.

\section{Task 3. Experiments in Practical Combustion Systems}

This represents a parallel effort to investigate and document the importance of thermal history effects on char structure and reactivity in well-controlled and characterized coal flames. Comparative experiments will be carried out on two reactor facilities with widely varying flame type and the properties and reactivities of the chars characterized.

\section{Project Team}

The project involves three universities (Brown, Ohio State, and Boston University), in order to couple engineering experts in coal combustion and carbon science (at Brown and Ohio State) with research groups in the pure sciences specializing in modern computational chemistry (OSU) and in solid state physics (BU). The multidisciplinary team will apply modern scientific tools to the challenging technological problem of linking char combustion behavior with coal properties and processing conditions.

The project is supported by an advisory panel assembled from industry, academia, and the national laboratories with a wide range of expertise. The panel members are:

\author{
Hamid Farzan \\ Alan Kerstein \\ Harry Marsh \\ Arun Mehta \\ Richard McCreery \\ Nsakala Nsakala \\ Stuart Daw
}

Babcock and Wilcox Co

Sandia National Laboratories

University of Alicante

Electric Power Research Institute

Ohio State University

ABB Combustion Engineering

Oak Ridge National Labs 


\section{PROGRESS THIS PERIOD}

\section{Brief Summary of Activities}

This quarter, our work on carbon structure continued. A combination of hot stage in situ and ex situ polarized light microscopy was used to identify the preferred orientational of graphene layers at gas interfaces in pitches used as carbon material precursors. The experiments show that edge-on orientation is the equilibrium state of the gas / pitch interface, implying that basal-rich surfaces have higher free energies than edge-rich surfaces in pitch. This result is in agreement with previous molecular modeling studies and TEM observations in the early stages of carbonization. The results may have important implications for the design of tailored carbons with edge-rich or basalrich surfaces.

In the computational chemistry task, we have continued our investigations into the reactivity of large aromatic rings. The role of $\mathrm{H}$-atom abstraction as well as radical addition to monocyclic aromatic rings has been examined, and a manuscript is currently being revised after peer review. We have also shown that $\mathrm{OH}$ radical is more effective than $\mathrm{H}$ atom in the radical addition process with monocyclic rings. We have extended this analysis to $\mathrm{H}$-atom and $\mathrm{OH}$-radical addition to phenanthrene. Work on combustion kinetics focused on the theoretical analysis of the data previously gathered using thermogravametric analysis.

\section{Detailed Discussion of Progress}

\section{Section 1: Surface Anchoring in the Formation of Carbon Structure}

Previous reports have described results of new research on the development of crystalline order in coal chars and other carbon materials. The work to date has been concerned with order in bulk phases. Ordering at surfaces, however, is also an important aspect of carbonization. The structure of carbon materials is influenced by the presence of gas interfaces, both at the external surface of the carbon body, and in internal bubble cavities. In composites, structure is influenced by ordering at the interface of the second solid phase, which may be a fiber, ceramic or metallic particle, or, in the case of coal technologies, a solid mineral grain. The arrangement of graphene layers at surfaces can influence the reactivity (by determining the ratio of edge to basal sites at the gas interfaces), and the interfacial energy and binding characteristics in composites. It also determines the proper boundary condition for mesophase pitch flow problems, such as those that describe fiber spinning.

The surface alignment of elongated molecules, either rods or disks, is a fundamental property of a liquid crystal. The "surface anchoring" is characterized by a preferred angle (the "easy axis") and a free energy associated with rotation away from that axis. Surface anchoring angles and energies have been determined experimentally for many liquid crystal / surface combinations. There has not, however, been systematic study of the surface anchoring properties of carbon material precursors. Work was undertaken this quarter to systematically determine the favored alignment of graphene layers at gas interfaces in the early stages of carbonization. The work described below was funded jointly by the current project and the NSF CAREER program in the thermal sciences, as it has direct applicability to a variety of carbon material systems and thus the goals of both programs at Brown. 
In this work a hot-stage polarizing microscope with long-focal-length optics (see Fig. 1.1) was used to obtain real-time in situ video sequences of the free surface of pools of molten pitch. The pitch was AR-3X15-1 from Mitsubishi Chemical, supplied courtesy of Professor Dan Edie at Clemson University. Mesophase pitch was chosen as a model substance rich in high-molecular-

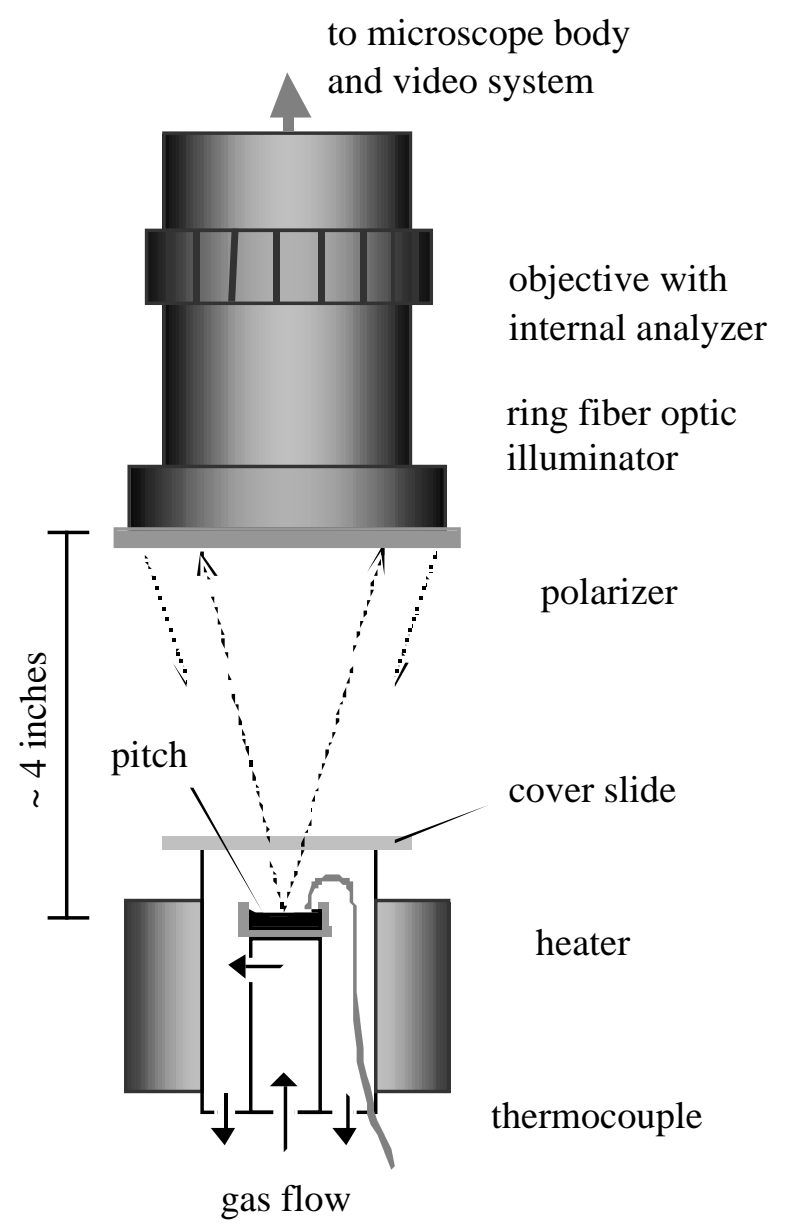

weight PAH with high fluidity, allowing characterization of the equilibrium surface anchoring properties. The mesophase pitch was contained in glass crucibles at $200-400{ }^{\circ} \mathrm{C}$ inside a custom designed helium-purged heated cell. Ex situ polarizing microscopy was also used to examine free surfaces and crosssections of quenched pitch samples at the end of the hot stage experiments.

Figure 1.1

Sketch of the long-focal-length microscope used for in situ hot-stage video imaging of molten mesophase pitch. The long focal length allows observation of a free surface (pitch / He interface) not in contact with a glass cover slide.

\section{Results}

Images of the free surface of the pitch pools (not shown) show classical liquid crystal texture with well-defined flow domains and sharp disclination lines. The strong optical anisotropy of this surface suggests a significant edge-on component of the surface layer orientations. (Note that strict face-on alignment would produce an isotropic surface.)

Strong evidence for near perpendicular edge-on orientation is seen in optical micrographs of polished cross-sections (Fig. 1.2 and 1.3). Figure 1.2 shows the gas/pitch interface at the free (top) surface in a polished cross-sections of pools of pitch, while Fig. 1.3 shows the gas / pitch interface at the interior surface of a bubble cavity with the pitch pool. Figure 1.2 shows various flow domains beneath the free surface of a pitch pool. When the flow domains consist of layers perpendicular to the free surface, the domain color extends fully to the interface. When, however, the flow domains consist of layers parallel to the surface, the domain switches to a surface film 
Figure $1.2 \quad$ Black-and-white reproduction of a color, polarized optical micrograph of a polished cross-section of quenched mesophase pitch. In the original, the dark regions are blue and the light regions are yellow. Flow domains with perpendicular alignment (white layer pairs on dark field) extend fully to the free surface. In contrast, flow domains with parallel alignment (dark layer pairs on light field) stop short of the free surface, giving way to edge-on surface films.

The optical system uses crossed polars with retarder plate. Layer orientations shown were deduced from the color original following calibrating of the optical system using the polished edges of Ticonderoga graphite flakes.

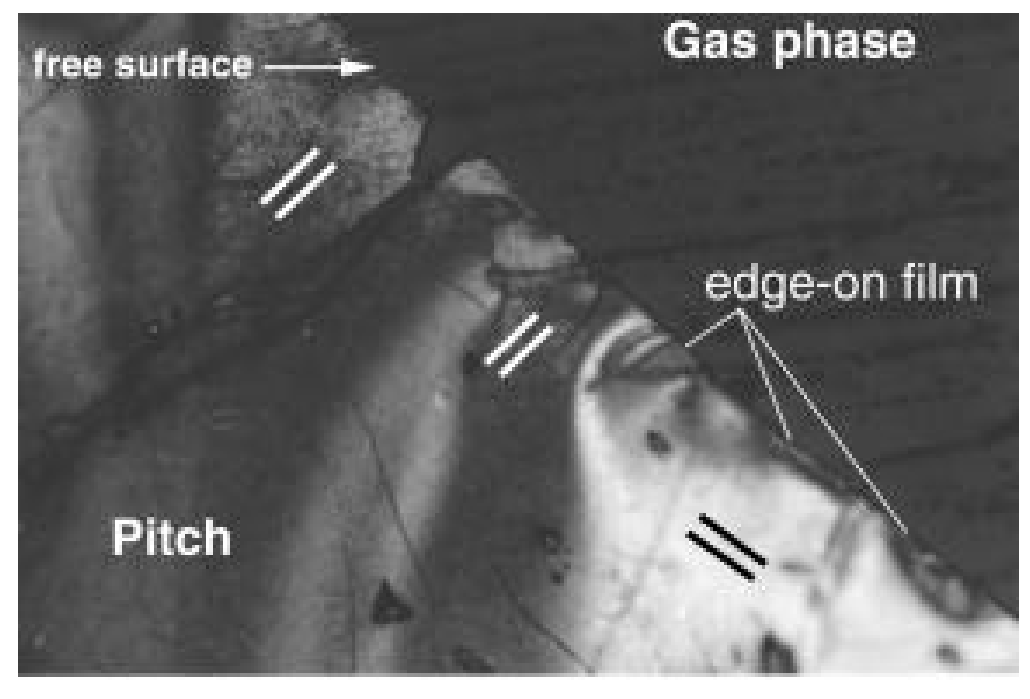

In Figure 1.3, layer alignment near cavities is seen to be parallel (face-on), except for edge-on alignment in a thin film of micrometer dimensions right at the cavity surface. It is believed that the stretching and drainage of the membranes surrounding the bubbles causes parallel alignment by a flow mechanism, while the true surface equilibrium state is the opposite (edge-on) and seen only in thin surface films.

\section{Discussion}

Overall the optical microscopy clearly demonstrates preference for near-perpendicular orientation of the polyaromatic mesogens at free surfaces (layers edge-on at gas/pitch interfaces). This trend has been pointed out previously by White et al. [1982] for the particular case of mesophase spheres that intersect the free surface in two-phase pitches, but is contrary to the orientation of graphene layers in many carbon materials, as will be discussed later. Our interpretation of this finding is as follows.

We have postulated that the equilibrium nanostructure in a mesophase-containing pitch is given by minimization of:

$$
\mathrm{G}=\sum\left(\mathrm{n}_{\mathrm{i}} \mathrm{g}_{\mathrm{i}}^{\mathrm{o}}\right)+\mathrm{G}_{\text {mix }}+\mathrm{G}_{\text {orient }}+\mathrm{G}_{\text {surface }}+\mathrm{G}_{\text {elastic }}
$$

in the absence of flows and external fields [Hu and Hurt, 2000]. In this expansion, $\mathrm{g}_{\mathrm{i}} \mathrm{O}$ represent the standard free energies of formation of the polycyclic aromatic building blocks as single- 


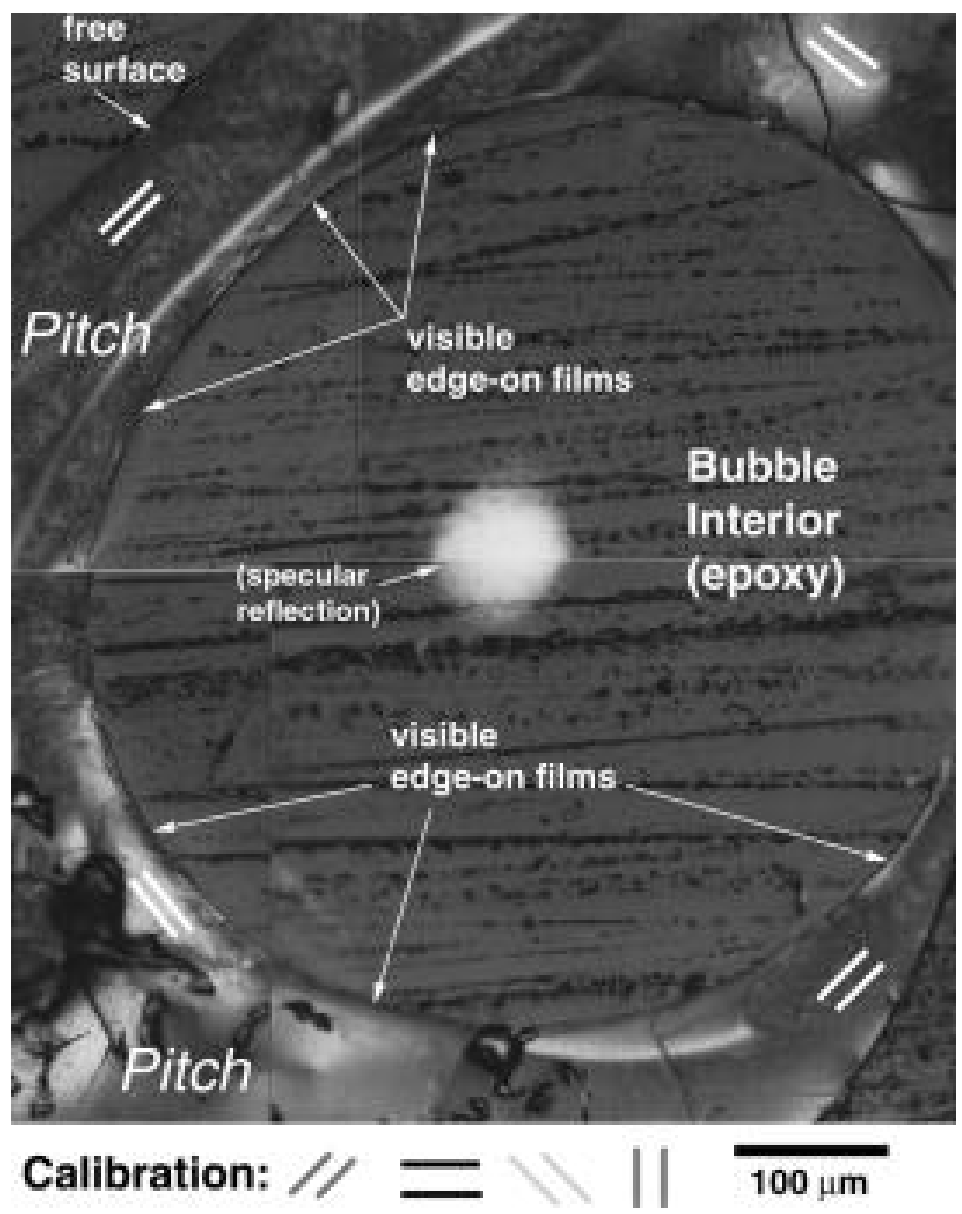

Figure 1.3. Optical micrograph of cross-section of quenched mesophase pitch, showing the free surface (pitch / He interface) and regions around an internal bubble. Layer orientations shown in the key were obtained by calibration using polished edges of Ticonderoga graphite flakes.

component isotropic condensed phases; $\mathrm{n}_{\mathrm{i}}$ represent the mole numbers; and the remaining terms are contributions to the system free energy derived from mixing, orientational order, surface or interfacial energies (including orientational effects at the surface), and elastic strain due to bend or splay in the ordered phase.

This report deals with the interfacial term, $\mathrm{G}_{\text {interface, }}$ which for gas / pitch interfaces is equivalent to the surface tension, $\gamma$. The overall interfacial tension consists of isotropic and anisotropic components:

$$
\mathrm{G}_{\text {interface }}=\gamma=\gamma_{o}+\mathrm{W}(\theta)
$$

where $\theta$ is the angle of departure of the directer from the easy axis and $\mathrm{W}(\theta)$ is a positive function. For discotic, the director is a unit vector perpendicular to the aromatic planes. The preferred surface 
orientation, $\theta$, is that which minimizes $\mathrm{G}$ in Eq. 2 (and thus in equation 1.) By this principle, the microscopic observations imply that the surface tension is lower for an edge-on surface than for a surface consisting of faces, or basal planes; i.e. $\gamma_{\text {edge }}<\gamma_{\text {basal, }}$ or that the easy axis places the director perpendicular to the unit vector defining the surface. Note that crystalline graphite samples show the opposite behavior, with surface energies higher at edge faces than basal faces. These energies reflect unsaturated covalent bonds at edge sites, however, and are not representative of hydrogen-saturated polyaromatic clusters. These clusters interact by physical forces, and theoretical and experimental evidence is strongly in favor of face-to-face association as the dominant interaction (see below).

A number of other observations in the literature provide indirect support for the finding that $\gamma_{\text {edge }}<$ $\gamma_{\text {basal }}$ in pitch. First, there is evidence that face-to-face association is the dominant interaction between polyaromatic clusters in the early stages of carbonization. The first structures to form in the early stages of carbonization are face-to-face associated clusters of 2-4 disk-like polyaromatic clusters, or basic structural units [Oberlin, 1965]. Also, mesophase has been observed by HRTEM to possess a partial columnar nature, again suggesting strong face-to-face association. Molecular modeling calculations by Vorpagel and Lavin [1992] on the fully-condensed PAH coronene, circumcoronene, yield binary interactions that are much stronger in the face-to-face or shifted-stack configuration than in the edge-to-edge configuration. Each of these results likely reflects the high relative strength of $\pi-\pi$ interactions. Edge-on alignment at the gas interface maximizes the internal $\pi-\pi$ interactions (it internally satisfies all faces) and thus leads to the lowest surface free energy. Secondly, aromatic compounds tend to have higher surface tensions than aliphatic compounds, and thus the aromatic basal-rich surfaces may be expected to have higher tensions than edge-rich surfaces, which are partially aliphatic due to short aliphatic side chains at the periphery of polyaromatic mesogens.

Finally, a word should be said about graphene layer orientations at interfaces in fully carbonized samples. High-temperature heat treatment causes extensive dehyrogenation and aromatization reactions that lead to edge-to-edge coalescence of the layers. The thermodynamic driving force for these chemical reactions far exceeds the thermodynamic driving forces for the alignment under the physical forces that dominate in fluid pitch [Vorpagel and Lavin,1992]. Thus graphite edge energies are higher than basal energies, due to unsaturated bonds at edge sites. Under conditions of high chemical reactivity, therefore, the equilibrium structures are those that allow satisfaction of reactive edge sites, which in the presence of most surfaces (such as a gas interface) is the face-on configuration. Thus there is both a physical equilibrium state for hydrogen-rich pitches in which layers are edge-on at gas interfaces, and an opposing chemical equilibrium state in hydrogendeficient carbon materials in which layers are face-on at gas interfaces. More work is needed to determine under what conditions the equilibrium edge-on films in pitch remain in a frozen metastable state during complete carbonization, and under what conditions they are capable of rearranging to the lower free energy face-on configuration during dehydrogenation and complete carbonization. Further experiments are also underway to systematically characterize the equilibrium anchoring at various solid/pitch (vs. gas/pitch) interfaces. 


\section{References for Section 1}

White, J.L., Buechler, M., Ng, C.B., Carbon, 1982, 20, 536.

Oberlin, A., in Chemistry and Physics of Carbon, Volume 22, Marcel Dekker, Inc. New York, 1965.

Vorpagel, E.R., and Lavin, J.G., Carbon , 1992, 30, 1033.

Hurt, R.H., Crawford, G.P., Shim, H.-S., "Equilibrium Structure of Primary Soot Particles," accepted for publication in the Proceedings of the Twenty-Eighth Symposium (International) on Combustion, The Combustion Institute, Pittsburgh, 2000.

Hu, Y., Hurt, R.H., "Thermodynamics of Carbonaceous Mesophase II: General Theory for NonIdeal Solutions," submitted to Carbon, January, 2000.

\section{Section 2: Progress in Computational Chemistry}

In this quarter, we have continued our investigations into the reactivity of large aromatic rings. In a recent quarterly report, we described some initial studies into $\mathrm{H}$-atom and $\mathrm{OH}$-radical addition to some polycyclic aromatic rings. These calculations are quite demanding due to the size of the chemical systems, but we have shown that density functional theory (DFT) methods[1] can be accurately applied to the study of $\mathrm{H}$-atom abstraction for monocyclic aromatic rings. [2] One attractive aspect of that study was that DFT methods are more computationally practical for the accurate consideration of large systems, such as the polycyclic aromatic hydrocarbons (PAHs) that are present in coal.[3]

The role of $\mathrm{H}$-atom abstraction as well as radical addition to monocyclic aromatic rings has been examined, and a manuscript is currently being revised after peer review.[4] H-atom abstraction is of greater relevance to organic substrates under combustion temperatures, but radical addition to the monocyclic rings is a viable pathway for certain ring sizes, such as 5-membered rings. In this quarter, we have extended these studies on monocyclic rings to polycyclic aromatic rings in order to examine the relative reactivity of edge $v s$ bridgehead carbon atoms in some planar PAHs. We have continued to use the B3LYP/6-31G(d) level of theory[5] to probe the activation barriers and the products of radical addition to a variety of PAHs. We have focussed on completing some of the initial studies of $\mathrm{H}$ and $\mathrm{OH}$ chemistry with some different PAHs.

Previously, we reported bond dissociation enthalpies (BDEs, $\Delta \mathrm{H}_{298}, \mathrm{kcal} / \mathrm{mol}$ ) for some planar PAHs, [2] and we noted that, in general, the C-H BDE is not significantly affected by the size of the $\mathrm{PAH}$ and all of the $\mathrm{C}-\mathrm{H}$ bonds in these planar aromatic networks are very similar. We have examined the role of $\mathrm{H}$-atom abstraction by $\mathrm{H}$ atom (a viable reactive intermediate present in the radical pool) with naphthalene, and the results are presented in Figure 1. In the most recent quarter, we calculated $\mathrm{H}$-atom addition to naphthalene, and for comparison, the data are presented in Figure 2. The activation barriers (at $298 \mathrm{~K}$ ) are significantly lower for $\mathrm{H}$-atom abstraction than they are for addition to the naphthalene ring; however, at high temperatures, the abstraction process will be favored. From Figure 1, it is obvious that there is very little difference between H-atom abstraction for the two unique $\mathrm{C}-\mathrm{H}$ bonds in naphthalene. 
We have also shown that $\mathrm{OH}$ radical is more effective than $\mathrm{H}$ atom in the radical addition process with monocyclic rings.[4] We have extended this analysis to $\mathrm{H}$-atom and $\mathrm{OH}$-radical addition to phenanthrene. Figure 3 shows that with phenanthrene, $\mathrm{H}$-atom addition has an activation barrier that is greater than $8 \mathrm{kcal} / \mathrm{mol}\left(\Delta \mathrm{G}_{298}\right)$ for the different positions on phenanthrene. In Figures 4 and 5 , we have calculated the analogous transition states for $\mathrm{OH}$ radical addition to phenanthrene and the reactions with $\mathrm{OH}$ radical are more facile and have lower activation barriers, in general. The reactivity at the edge atoms is significantly $(\sim 10 \mathrm{kcal} / \mathrm{mol})$ more facile than any reactions at the bridgehead carbon atoms.

Thus it appears that reactions on the edge atoms will always be more facile than reactions on the bridgehead positions. Furthermore, the relative reactivity of different radicals are similar between the monocyclic aromatic rings and the polycyclic systems.

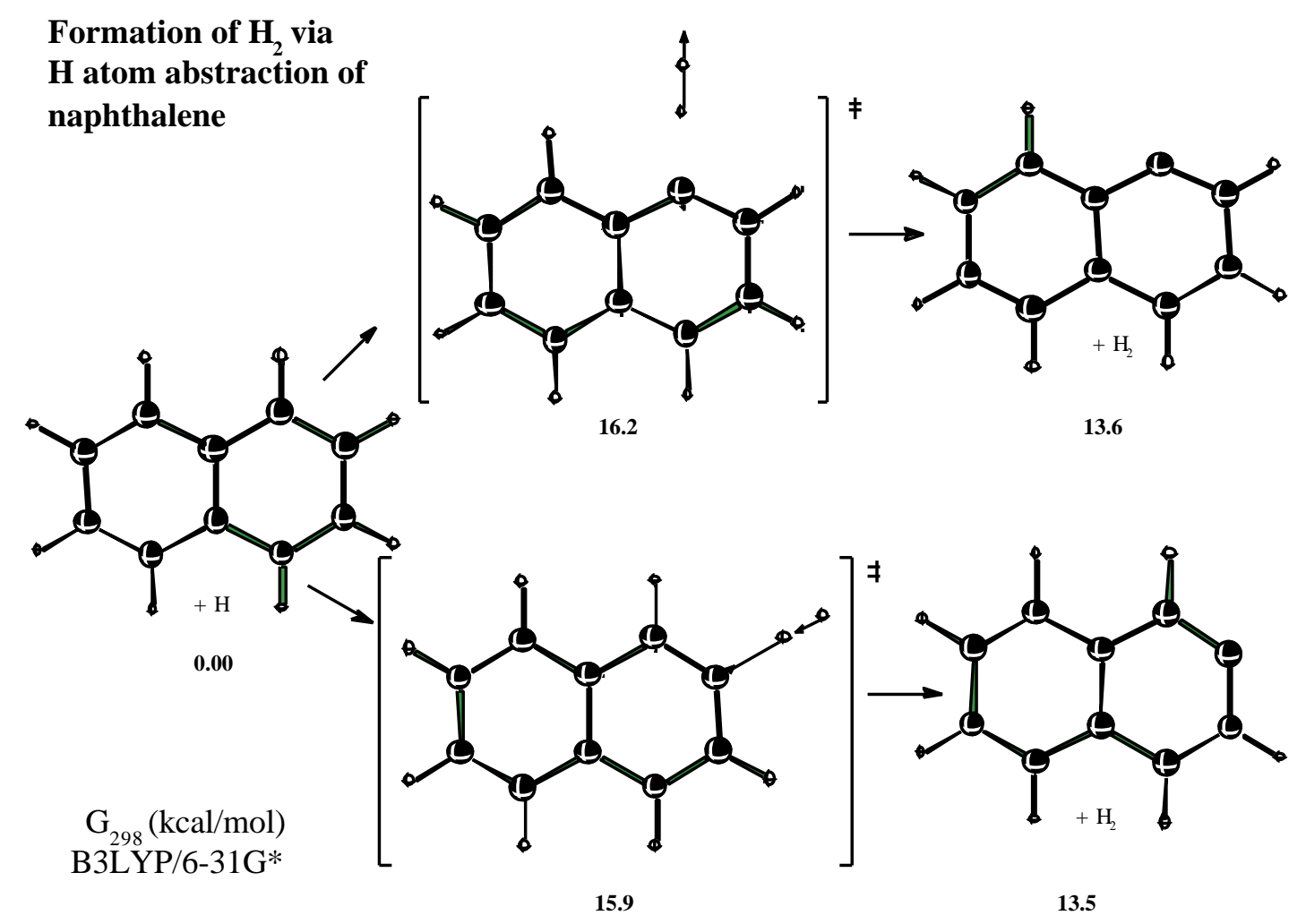

Figure 2.1. H-atom abstraction transition states and products with naphthalene $\left.\left(\Delta \mathrm{G}_{298}, \mathrm{kcal} / \mathrm{mol}\right), \mathrm{B} 3 \mathrm{LYP} / 6-31 \mathrm{G}(\mathrm{d})\right)$. 


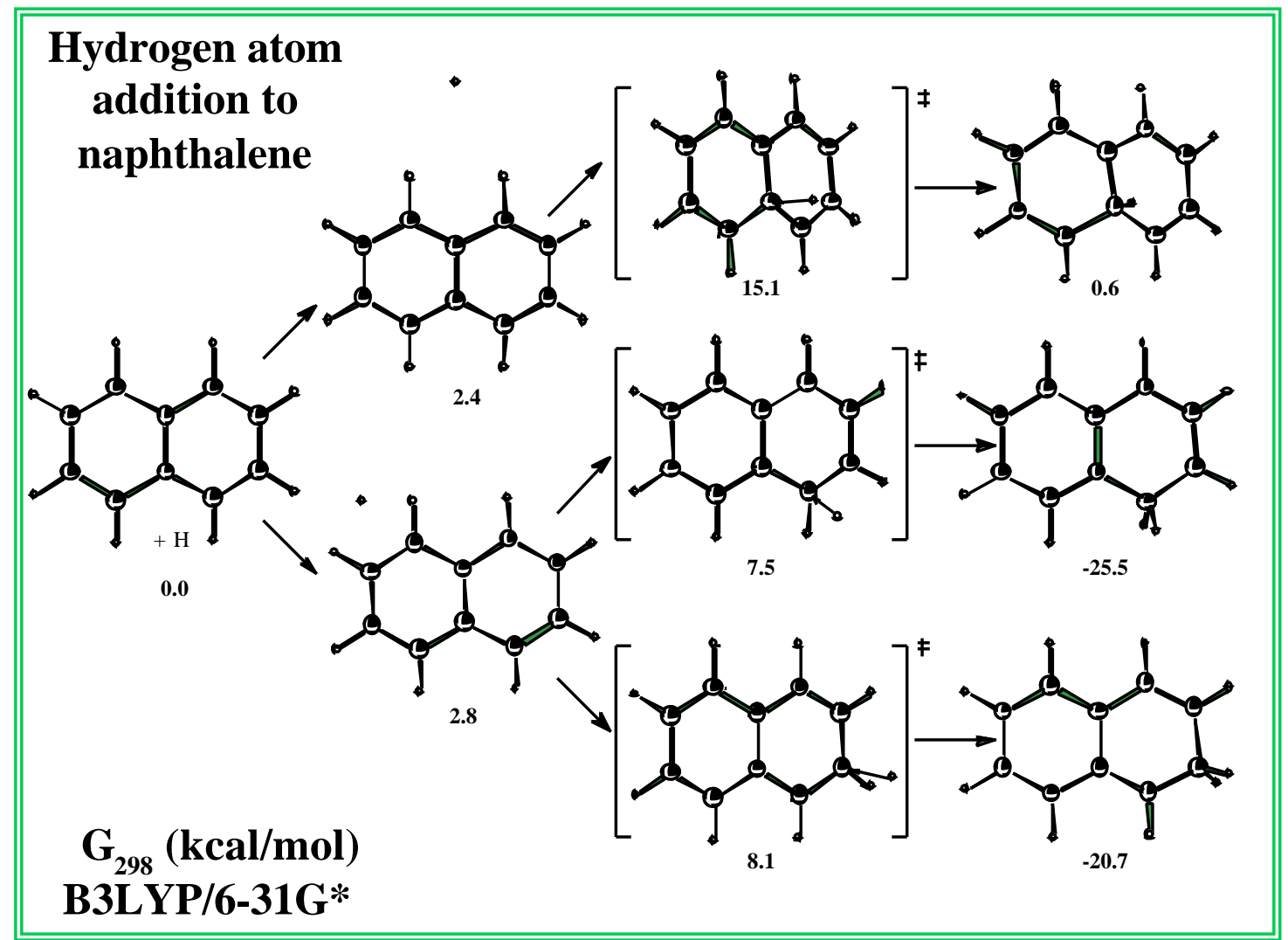

Figure 2.2 Comparison of $\mathrm{H}$-atom reactivity with the edge and bridgehead carbon positions of naphthalene $\left(\Delta \mathrm{G}_{298}, \mathrm{kcal} / \mathrm{mol}\right)$ at the B3LYP/6-31G(d) level. 


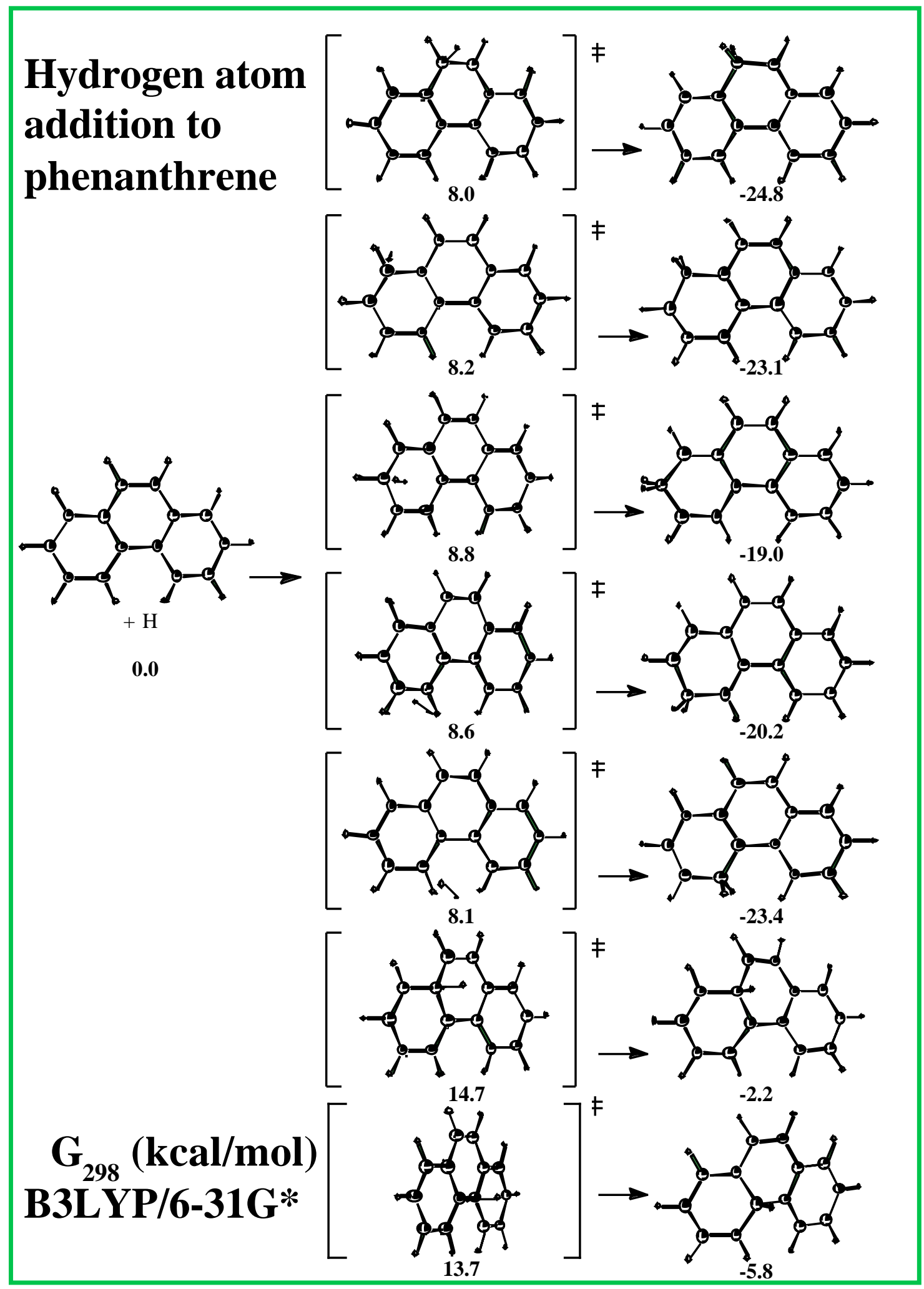

Figure 2.3. Comparison of $\mathrm{H}$ atom reactivity with the edge and bridgehead carbon positions of phenanthrene $\left(\Delta \mathrm{G}_{298}, \mathrm{kcal} / \mathrm{mol}\right)$ at the B3LYP/6-31G(d) level. 


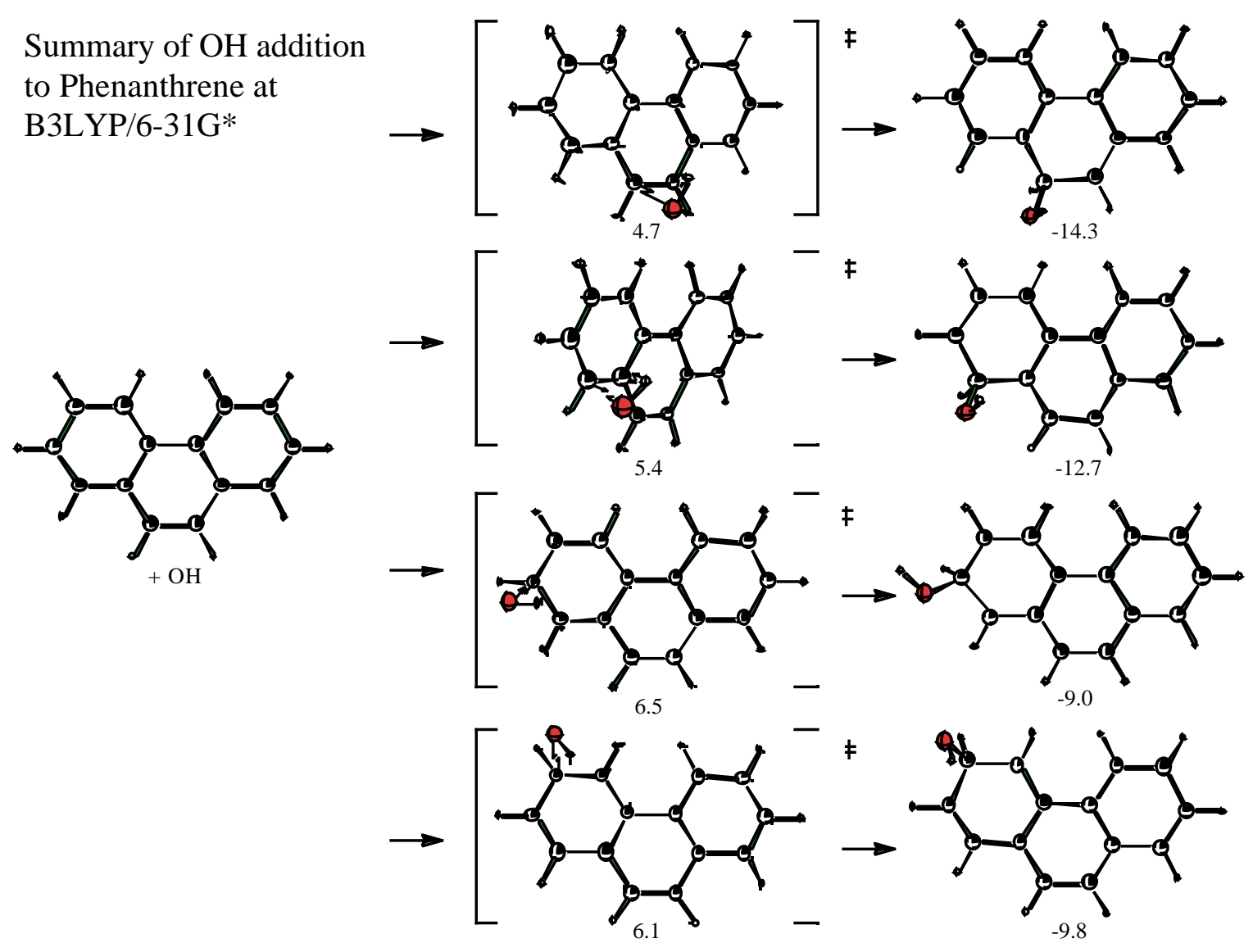

Figure 2.4. Comparison of $\mathrm{OH}$ radical reactivity with some of the edge and bridgehead carbon positions of phenanthrene $\left(\Delta \mathrm{G}_{298}, \mathrm{kcal} / \mathrm{mol}\right)$ at the B3LYP/6-31G $(\mathrm{d})$ level. 
Summary of $\mathrm{OH}$ addition to Phenanthrene at B3LYP/6-31G*

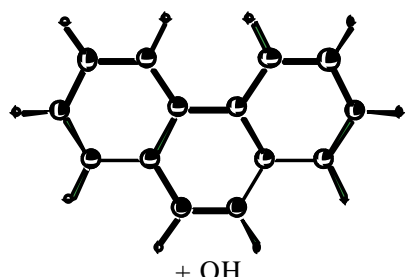

0.0
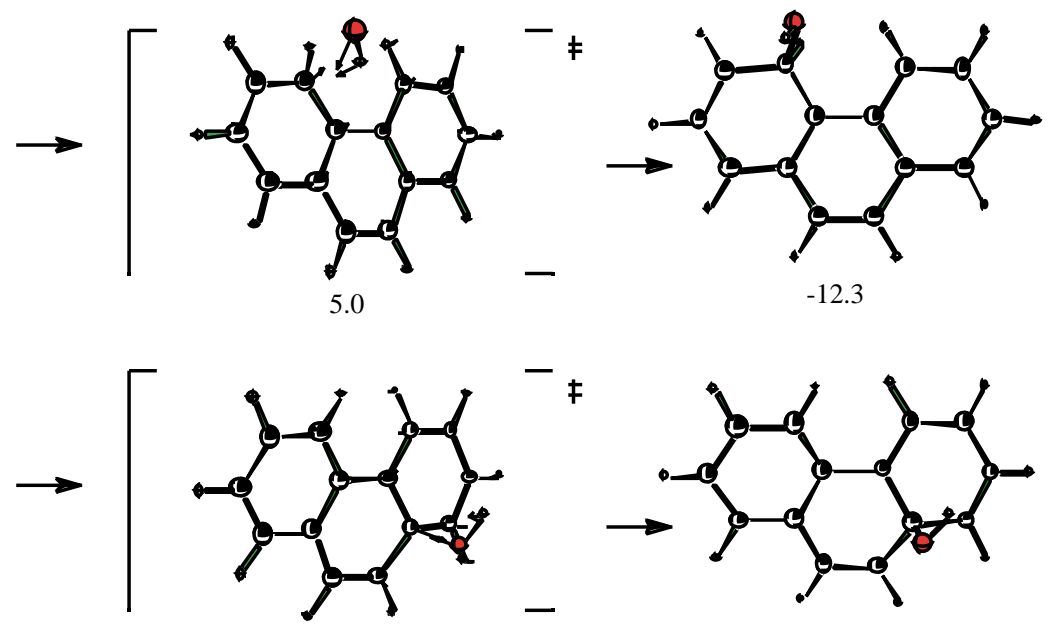

14.3

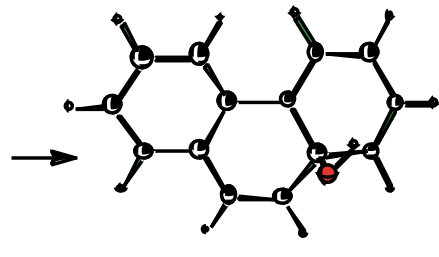

7.4

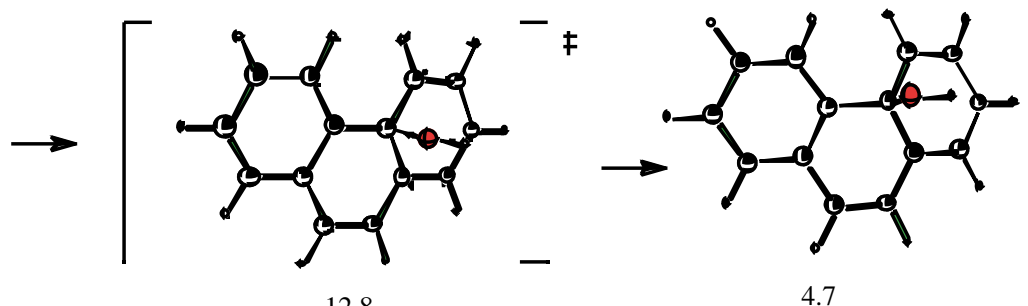

Figure 2.5. Comparison of $\mathrm{OH}$ radical reactivity with some of the edge and bridgehead carbon positions of phenanthrene $\left(\Delta \mathrm{G}_{298}, \mathrm{kcal} / \mathrm{mol}\right)$ at the B3LYP/6-31G(d) level.

\section{References for Section 2}

[1] Labanowski, J. W.; Andzelm, J. Density Functional Methods in Chemistry; Springer: New York, 1991. Parr, R. G.; Yang, W. Density Functional Theory in Atoms and Molecules; Oxford University Press: New York, 1989.

[2] Barckholtz, C.; Barckholtz, T. A.; Hadad, C. M. J. Am. Chem. Soc. 1999, 121, 491.

[3] Lyon, L. B.; Hill, G. R. Ind. Eng. Chem. 1962, 54, 36. Meyers, R. A. Coal Structure; Academic Press: New York, 1982.

[4] Barckholtz, C.; Hadad, C. M. J. Am. Chem. Soc., submitted for publication.

[5] Becke, A. D. J. Chem. Phys. 1992, 98, 1372. Miehlich, B.; Savin, A.; Stoll, H.; Preuss, H. Chem. Phys. Lett. 1989, 157, 200. Lee, C.; Yang, W.; Parr, R. G. Phys. Rev. B 1988, 37, 785. 


\section{Section 3: Combustion Experiments with Contrasting Flame Types}

Work performed this past quarter has focussed on theoretical analysis of the data previously gathered. Thermogravametric analysis (TGA) is a well-established and widely used technique for determining the characteristics of coal. The TGA measures weight loss as a function of time and temperature. The overall description of the behavior of the char in the TGA is a brief period of time during which the particle may physically absorb oxygen onto the surface. When the temperature is high enough, reaction commences in Zone II and continues for a few minutes, at most. Then the kinetics shift to Zone I and reaction continues, reaching final burn-out, for a period of time normally longer than the Zone II reaction.

In general, the TGA analysis involves determining the following: the point of inflection of the mass loss vs. time curve, ascertained by numerically differentiating the data and identifying the maximum of this derivative; the temperature at point of inflection, $\mathrm{T}_{\text {inflec }}$; the time at point of inflection, $\mathrm{t}_{\text {inflec }}$; and the percent ash in the sample identified as the residual weight of sample remaining after the TGA is complete. The percent ash in the sample is used to calculate the combustion efficiency and the rate of change of mass with time is used to calculate the activation energy.

The TGA data were analyzed with the goal of producing a relative ranking of reactivity at each port of the furnace. Specifically, two methods were employed for this analysis. The first method involves calculating a parameter to quantify the reactivity, $\mathrm{R}=100(\mathrm{H} / \mathrm{t})$, where $\mathrm{H}\left[\mathrm{sec}^{-1}\right]$ is the height of the peak of the maximum derivative of weight loss with respect to time and $t$ is the time [sec] corresponding to this peak. Although no kinetic parameters are extracted through the use of this method, it does rank the thermogravimetric reactivity of the chars and coal in the combustion process. The general procedure was to numerically differentiate the plots of weight loss vs. time and to use the Eqn. 1 to create plots of the relative reactivity vs. port.

The second method invokes the assumption that the slope of the weight loss vs. time curve is (approximately) constant. This method also gives a relative ranking of the chars with the added advantage that it allows for the extraction of activation energies.

The most common analytical procedure involves taking the first derivative of weight change with time and normalizing this quantity against either is initial mass or the mass at the time of the measurement. This quantity is defined as the reactivity at a specified temperature, and, in general, can be written as

$$
\mathrm{R}_{\mathrm{s}}=(\mathrm{dm} / \mathrm{dt})\left(1 / \mathrm{m}^{\mathrm{z}}\right)
$$

where $\mathrm{m}$ is the mass of the sample at the point the derivative is taken and $\mathrm{z}$ accounts for internal reaction. Results of the Thiele analysis indicate that $z=2 /(3+\alpha)$. At the limits where $\alpha$ is 0 or approaches infinity, $\mathrm{z}$ is $2 / 3$ and 0 , respectively. Normally, $\mathrm{z}$ is set to one; although, this implies that $\alpha$ is negative, which is theoretically impossible as it implies a negative value of internal surface area per unit volume, effective velocity constant, gas density, or diffusion coefficient.

The TGA operates in temperature range that is low enough to allow for relatively fast diffusion and adsorption of oxygen. The rate-limiting step is desorption of the oxygen complexes from the surface. Thus, the appropriate form of the rate expression is 


$$
1 / \mathrm{R}_{\mathrm{s}}=1 / \varepsilon \mathrm{k}_{\mathrm{d}}
$$

or

$$
\mathrm{R}_{\mathrm{s}}=\varepsilon \mathrm{k}_{\mathrm{d}}{ }^{\circ} \exp \left(-\mathrm{E}_{\mathrm{d}} / \mathrm{RT}\right)
$$

Plotting this equation in Arrhenius form should produce a straight line of slope (- $\left.E_{d} / R\right)$ and intercept that is a function of $k_{d}{ }^{\circ}$. Extraction of the activation energy is the main goal of this method; knowledge of the activation energy allows for a systematic ranking of the reactivity of the samples collected from different points in the furnace. Referring to Eqn. 1 and applying the chain rule,

$$
\mathrm{R}_{\mathrm{s}}=(\mathrm{dm} / \mathrm{dT})(\mathrm{dT} / \mathrm{dt})\left(1 / \mathrm{m}^{2}\right)
$$

where the rates of change of temperature with time and mass with temperature, $\mathrm{dT} / \mathrm{dt}$ and $\mathrm{dm} / \mathrm{dT}$, are both assumed to be constant. Substituting into Eqn. 3 and taking the natural log of each side,

$$
\ln \left(1 / \mathrm{m}^{2}\right)=\ln \left[\left(\mathrm{k}_{\mathrm{o}}\right) /(\mathrm{dm} / \mathrm{dT})(\mathrm{dT} / \mathrm{dt})\right]-\left(\mathrm{E}_{\mathrm{d}} / \mathrm{R}\right)(1 / \mathrm{T})
$$

Plotting $\ln \left(1 / \mathrm{m}^{2}\right)$ vs. (1/T) in Arrhenius fashion gives a straight line of slope (E / R) and intercept $\ln \left(\mathrm{k}_{\mathrm{o}}\right) /[(\mathrm{dm} / \mathrm{dT})(\mathrm{dT} / \mathrm{dt})]$. This technique eliminates any dependence on the rate of change of mass with time, $\mathrm{dm} / \mathrm{dt}$, and instead allows for an analysis based on the current sample mass.

The plot of mass loss vs. time generated by the TGA is a sigmoid; thus, there exists a point of inflection that occurs at the maximum derivative of weight loss with respect to time. It is not sufficient to assume that the slope of the weight loss vs. time curve is constant through the entire TGA weight loss vs. time curve; the magnitude changes at the point of inflection. To account for this property, all of the TGA data were further analyzed in the following manner. The point of inflection was identified and one line was fitted to the data above this point and another was fitted to the data below this point. The slope of each line was determined separately and two activation energies were extracted: one for the upper fit and one for the lower fit.

Magnitudes of the error bars were determined by randomly selecting points in the linear portion of the weight loss vs. time curve and determining the slopes corresponding to these random points. This procedure was repeated three times and, in conjunction with the results obtained using every point, standard deviations were computed; the error bars represent one standard deviation. Where the data set included multiple analyses performed on the same port, error bar magnitudes were obtained from the standard deviation of these multiple measurements.

The basic premise in this study is that the coal char is subjected to the two competing processes of annealing and burnout. The proposition is that: as the annealing progresses, the reactivity will drop. If the combustion process is very rapid, it will be completed prior to any significant annealing. However, if the annealing is very rapid, the reverse result is obtained: the initial combustion may be fast; but, the final burn-out may be significantly extended. The overall results do appear to support this premise. 


\section{$\underline{\text { Reactivity and Activation Energy }}$}

Plots of reactivity are in good agreement with those of overall activation energy. Examples of the plots of reactivity and activation energy as calculated for Run 1 are displayed in Figs. 1 and 2. A decreased reactivity can be expected to correlate with an increased activation energy. A correlation between the reactivity and activation energy is critical as it reinforces the validity of both methods of analysis. Although the magnitude of the reactivity calculated appears to have no fundamental meaning, it does serve as a basis for a relative ranking and, from this, the general trend is: if the temperature at the point of inflection is delayed, the char is less reactive. The values of activation mostly fall within the range of $20-50 \mathrm{kcal} / \mathrm{gmol}$, which is in the range reported in previous work by other investigators.

An important observation in this study is that the nature of the TGA weight loss vs. time curves evidently produces two separate regions in which the activation energy changes magnitude. As can be seen from Fig. 3, the activation energies below the point of inflection are, on average, twice the activation energies above this point. Figure 4 shows a correlation of the results with the best fit line of slope 1.2 and, this result is in good agreement with the existing theory of Zone kinetics; though, usually, transition from Zone II to Zone I occurs with falling, not with increasing temperature. The results obtained show the activation energy to halve with decreasing temperature. This apparent contradiction is resolved by considering the penetration depth of oxygen into the particle. As the particles begin to react in the TGA at the low temperatures the penetration depth is proportionately small and, since the rates of heating preclude any boundary layer diffusional or chemical kinetic limitations, the reaction is in Zone II. As the particle continues to react, the penetration depth increases and/or the particle size decreases until reaction finally reaches the center at which time the kinetics shift, by definition, into Zone I since the penetration depth in Zone I is equal to the particle radius. While in Zone I the reaction rate is slower and diffusion is able to maintain an increasingly uniform oxygen concentration throughout the particle.

\section{Combustion Efficiencies}

In the flame itself, combustion efficiencies are high, mostly over $90 \%$. In general, the results indicate that the bulk of the reaction is completed by the time the char reaches the first port. The results also show that the combustion efficiencies are well correlated with the excess air, showing the trend of decreasing excess air corresponding to decreasing combustion efficiency.

\section{Particle Size Distributions}

Particle size distributions show, in general, a shift from larger to smaller particles with increasing residence time. This is reasonable since the larger particles are reacting mainly at the surface and shrinking as they burn. The experiments performed with Pitt8a have the additional property of a broad size distribution in the raw coal and a narrow distribution in the chars. It appears that reaction not only shrinks the larger particles; but, also the smallest particles burn-out completely within the first few milliseconds. This postulate is well supported by detailed calculations reported by Misra in 1990. This area marks a point that is under continuing investigation. 


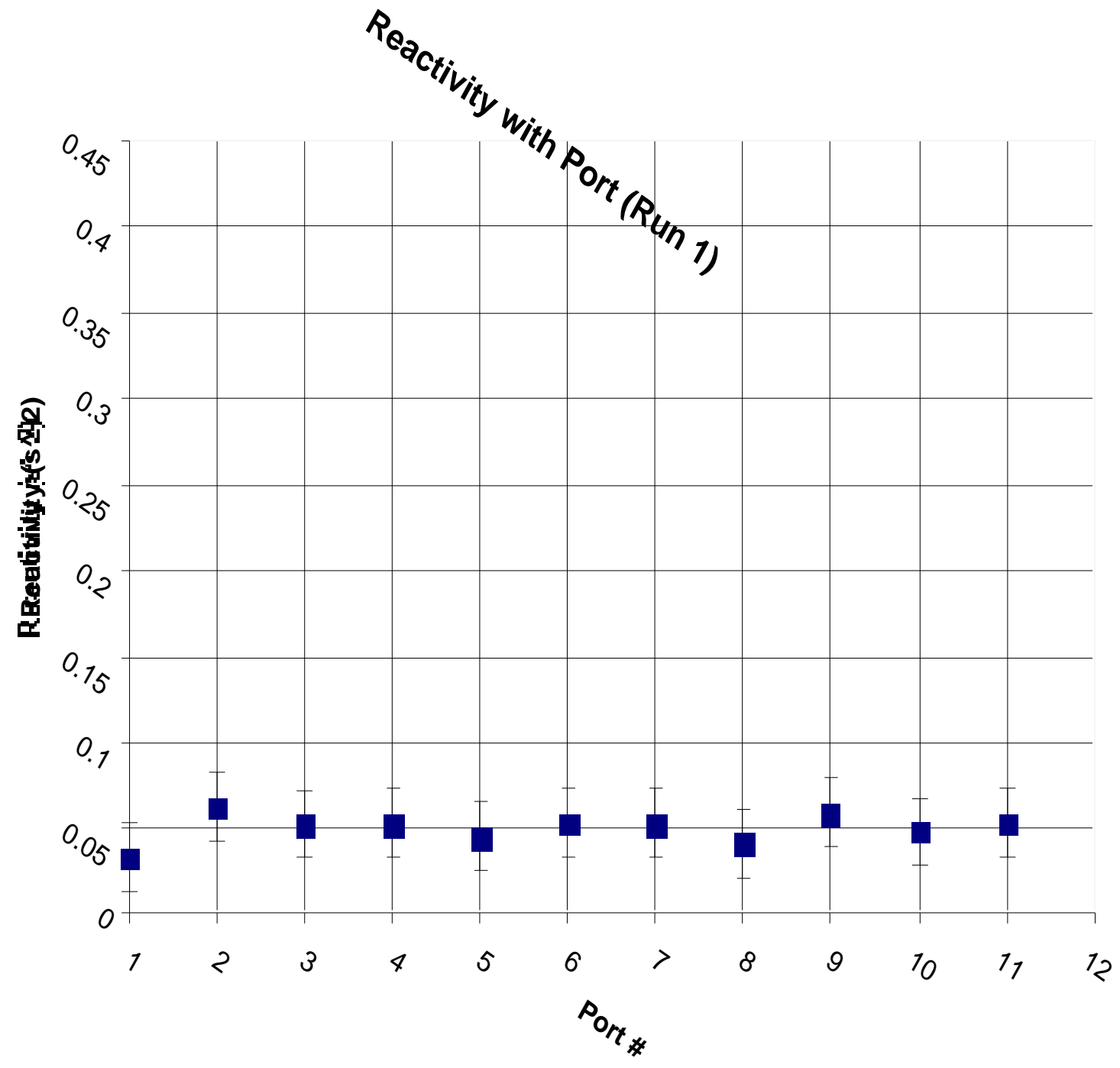

Figure 3.1. Reactivity as Height of Derivative Peak Divided by Time for Each Port (Run 1). 


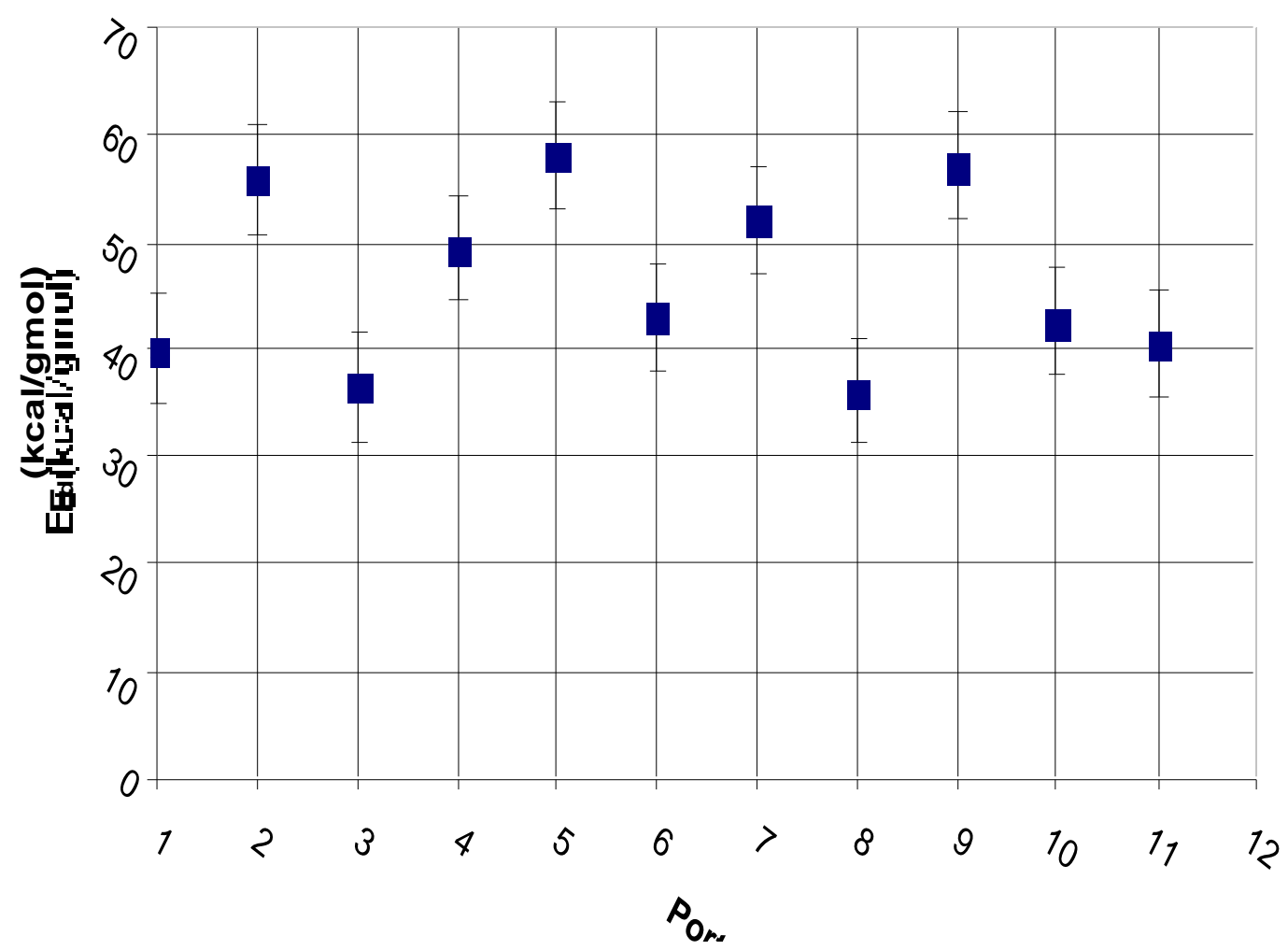

Figure 3.2. Variation of Overall Activation Energy for Each Port (Run 1). 


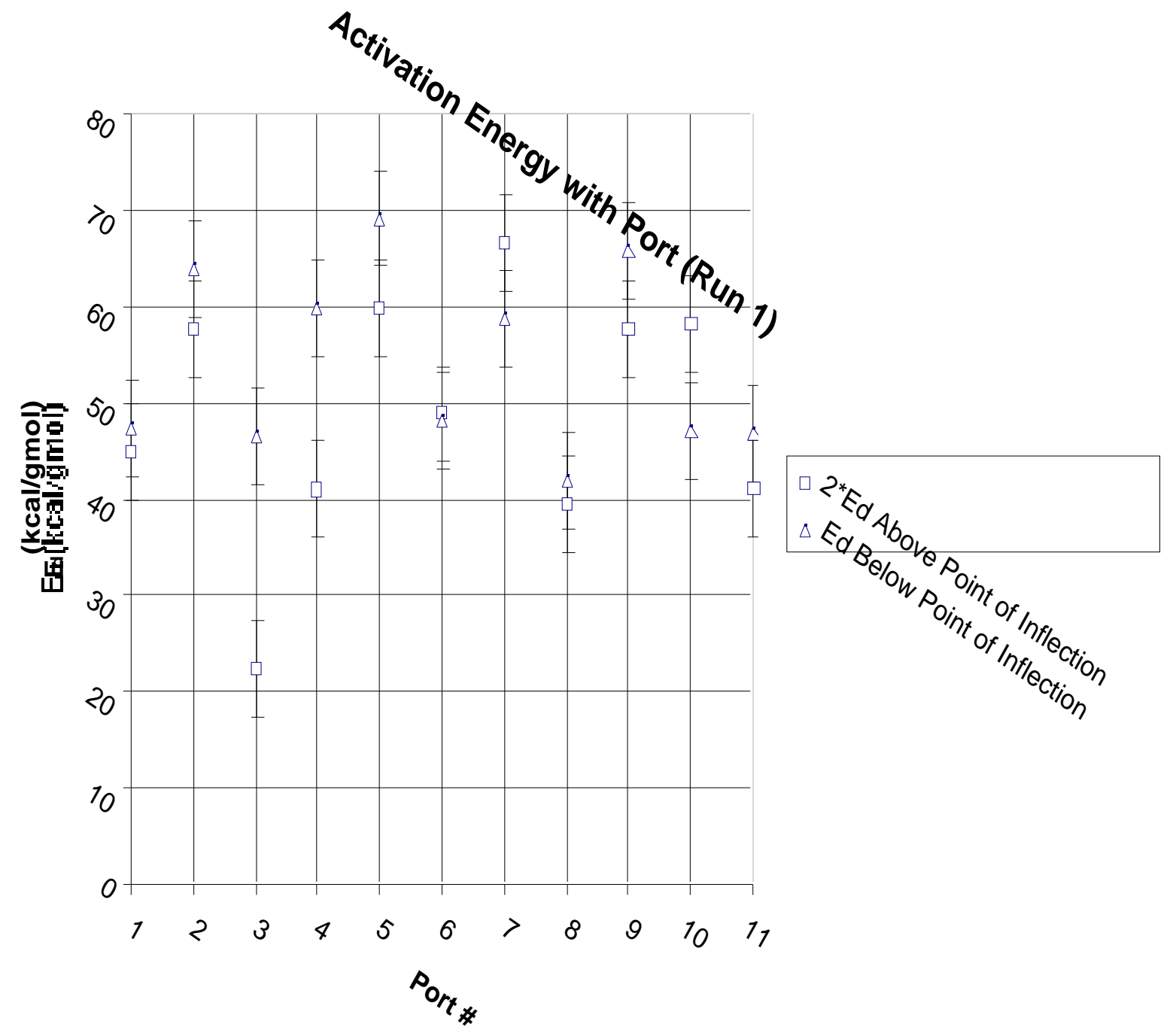

Figure 3.3. Variation of Activation Energy Calculated Separately for Data Above and Below Point of Inflection of TGA Weight Percent vs. Time Output (Run 1). 


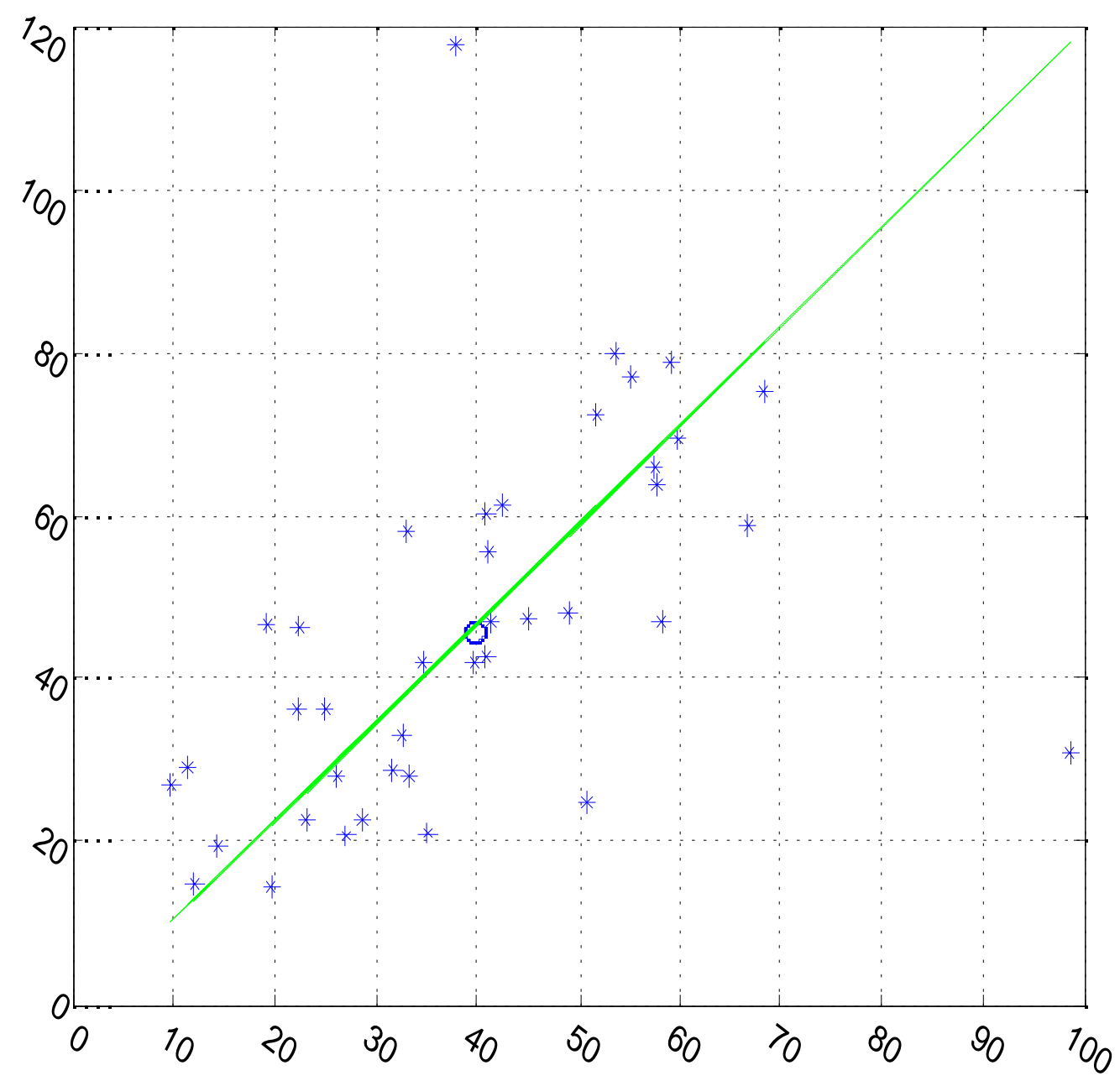

Figure 3.4. Correlation of Activation Energies Calculated Above and Below Point of Inflection. 